\title{
Prosthetic heart valve surgery and potential risk of developing Mycobacterium chimaera endocarditis
}

\author{
Authors: Shailesh Dalvi ${ }^{\mathrm{A}}$ and Paul Das ${ }^{\mathrm{B}}$
}

Public Health England (PHE) issued a guidance report on the management of Mycobacterium chimaera endocarditis following cardiac valve surgery. $M$ chimaera is a nontuberculous mycobacteria (NTM) belonging to mycobacterium avium complex (MAC). PHE has also recognised that $M$ chimaera has caused severe infections in a small proportion of patients who have had cardiac surgery. Investigations in the UK and internationally have implicated contaminated heater cooler units used for cardiopulmonary bypass. Prosthetic valve endocarditis (PVE) presents as the frequent and severe form of infective endocarditis (IE). The objective of this review is to discuss the role of clinicians in assessment, treatment and reassurance of all the patients who are recalled for clinical consultation following their risk of suspected $M$ chimaera infection after open-heart surgery.

KEYWORDS: Endocarditis, heart valve surgery, heart valve disease, heart valve disease follow-up

\section{Introduction}

Prosthetic valve endocarditis (PVE) presents as the frequent and severe form of infective endocarditis (IE).

Public Health England (PHE) has provided guidance on the management of Mycobacterium chimaera infection following cardiac valve surgery. This strain of M chimaera is a nontuberculous mycobacteria (NTM) belonging to mycobacterium avium complex (MAC), ${ }^{1,2,3}$

As per data available from the Society for Cardiovascular surgery in Great Britain and Ireland, 36,134 cardiovascular surgeries were carried out in 2013 alone. Of this, 17,630 coronary artery bypass grafting (CABG) and 18,504 involved valve and CABG surgeries. ${ }^{4}$

The objective of this review is to highlight the role of clinicians and transthoracic echocardiogram (TTE) in the follow-up of all patients who are recalled for clinical consultation following risk of suspected $M$ chimaera infection associated the open-heart surgery.

Authors: AST5 Cardiology, Glan Clwyd Hospital NHS, Wales Deanery,

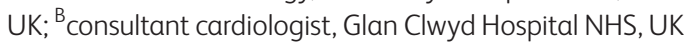

This review includes the nature of this infection and highlights the recommendations from authorised bodies such as PHE, the Centre for Disease Control and Prevention in the USA (CDC) and the European Centre for Disease Prevention and Control (ECDC). PHE has identified that $M$ chimaera causes severe infections in a small proportion of patients who have had cardiac surgery. The UK, alongside various international organisations, has implicated the contaminated heater cooler units (HCU) used during cardiopulmonary bypass surgery. The bacteria reaches the operating field through contaminated aerosol from the HCU water tanks.

\section{Discussion}

During the years 2014-15, a Swiss investigation team (Peter W Schreiber et al) identified that the source of $M$ chimaera was from the $3 \mathrm{~T} \mathrm{HCU}$ of cardiopulmonary bypass equipment, which transmitted the bacteria to the surgical site via contaminated water. $^{1,2}$

The ECDC subsequently published a report based on 52 cases of invasive cardiovascular infection which were caused by $M$ chimaera. In these cases, patients were identified to have previously undergone open-heart surgery in seven countries in Europe (France, Germany, Ireland, The Netherlands, Spain, the UK and Switzerland) since 2011. Similar cases have also been reported in the USA, Canada, Australia and Hong Kong.

The manufacturer of the 3T HCU introduced risk mitigation measures in September 2014, with enhanced cleaning and disinfection procedures. Isolation of NTM from 3T HCUs manufactured after September 2014 indicate that the risk has not yet been eliminated. ${ }^{1,5,7}$

The risk of infection by M chimaera and other pathogens associated with HCUs other than the $3 \mathrm{~T}$ remains unknown, but this remains a potential risk.

\section{Pathology and reported cases}

The US CDC has issued the following guidelines on diagnosing $M$ chimaera endocarditis.

'Patients have presented with a variety of clinical features including endocarditis, surgical site infection, abscess, and bacteraemia. Subsequent complications include renal insufficiency, hepatitis, splenomegaly, pancytopenia and osteomyelitis.'

Other presentations included granulomatous disease and have been misdiagnosed with sarcoidosis. ${ }^{8}$ 
PHE has recommended that the presentation and laboratory features of the disease can be similar to sarcoidosis. Clinical understanding of this infection continues to evolve. Clinical data in the following section are based on the first 25 UK cases, which included sternal wound infection, endocarditis and disseminated infection.

The incubation of $M$ chimaera is important to consider as it has a confounding effect on the diagnosis. M chimaera can have long latency periods following cardiothoracic surgery. The time between surgery and development of symptoms has been ranged from 2-58 months. ${ }^{1,2}$

Common clinical and laboratory features at presentation (>50\% of cases) were:

$>$ fever

$>$ malaise

$>$ weight loss

cough or shortness of breath

$>$ lymphocytopenia

$>$ hypoalbuminaemia

$>$ alkaline phosphatase >150 IU/L

$>$ C-reactive protein was noted to be less than $50 \mathrm{mg} / \mathrm{L}$.

Notable but less common features in the UK patient group at presentation included:

$>$ splenomegaly

eye involvement (eg choroidoretinitis)

$>$ thrombocytopenia and neutropenia.

Transthoracic echocardiography and transoesophageal echocardiogram (TOE) has been reported as normal in some patients at presentation who later went on to be diagnosed with prosthetic valve endocarditis, disseminated disease or aortic graft infection. Like any other infective endocarditis, a normal echocardiogram does not necessarily imply absence of infection.

PHE has set out guidelines in regards to investigating patients

for $M$ chimaera infections when associated with cardiopulmonary bypass.

1 Discuss all suspected cases with cardiology and infectious diseases specialists.

2 All patients should be investigated as per national guidelines for endocarditis. This includes pathology, imaging and microbiology for the common causes of the syndrome. M chimaera remains a rare cause of post-surgical infection.

3 All patients who have had exposure to bypass HCUs and who present with the relevant clinical syndromes should have microbiological investigations specifically directed towards M chimaera.

4 Patients with suspected or confirmed M chimaera surgical site sternal wound infection should have imaging to assess whether there is underlying deep infection.

\section{Microbiological investigation}

Mycobacterial culture is the essential investigation as it allows bacterial species identification and antibiotics susceptibility testing.

16S rRNA gene sequencing may be used as a supplementary test, but a negative result does not rule out infection.

It is important to inform the microbiologist when $M$ chimaera infection is suspected to ensure correct processing.

$>$ In case of suspected endocarditis: take three sets of mycobacterial blood cultures.
$>$ Any tissue or histology samples (eg valve tissue) should be tested by mycobacterial culture and $16 \mathrm{~S}$ rRNA gene sequencing in addition to the usual investigations.

> Wound infection: mycobacteria cannot be cultured from swabs, so tissue, bone or pus samples should be processed for mycobacterial culture.

> Patients with possible disseminated disease: take three sets of mycobacterial blood cultures and early morning urine for mycobacterial culture.

> Patients with pulmonary infiltrates: in addition to three sets of mycobacterial blood cultures, consider sending bronchoalveolar lavage +/- relevant biopsies for mycobacterial culture (essential) and $16 \mathrm{~S}$ rRNA gene sequencing.

In the first instance, clinical advice should be obtained from the local infection team. Infection teams requiring specialist advice should contact the nearest reference service.

In addition to $\mathrm{M}$ chimaera, $\mathrm{PHE}$ has described other infections potentially acquired from HCUs.

Other organisms such as Legionella and Stenotrophomonas maltophilia have been detected in HCUs and may pose a theoretical risk of transmission to patients and, in some cases, staff.

A further discussion of this is available in the infection control guidance:

www.gov.uk/government/publications/infections-associatedwith-heater-cooler-units-used-in-cardiopulmonary-bypassand-ecmo ${ }^{3}$

\section{Clinical management}

\section{Expert opinion on the diagnosis of M chimaera endocarditis}

Leading experts in echocardiography from London, Oxford and Cardiff have given their opinion that all patients who are recalled for screening and consultation should be offered routine TTE along with clinical examination and three sets of blood cultures as per PHE recommendations.

Although TTE has low sensitivity and specificity in detecting prosthetic valve endocarditis, it has provision to detect major valve defects such as regurgitation, paravalvular leaks. Normal TTE can be archived as a baseline study for future reference. Further TOE can be carried out if TTE is inconclusive.

From the patient's perspective, the initial TTE will provide reassurance with regard to the functional status of their valve.

\section{References}

1 Schreiber PW, Kuster SP, Hasse B et al. Reemergence of Mycobacterium chimaera in heater-cooler units despite intensified cleaning and disinfection protocol. Emerg Infect Dis 2016;22:1830-3.

2 European Centre for Disease Prevention and Control (ECDC). Invasive cardiovascular infection by Mycobacterium chimaera potentially associated with heater-cooler units used during cardiac surgery 2015 http://ecdc.europa.eu/en/publications/Publications/mycobacteriumchimaera-infection-associated-with-heater-cooler-units-rapid-riskassessment-30-April-2015.pdf. [Accessed 20 June 2018].

3 Public health England. Mycobacterium chimaera infections associated with cardiopulmonary bypass. Clinical guidance for secondary care. Version 1. PHE, 2017.

4 Blue book online: The Society for Cardiothoracic Surgery in Great Britain and Ireland http://bluebook.scts.org/ [Accessed 20 June 2018]. 
5 Sax H, Bloemberg G, Hasse B et al. Prolonged outbreak of Mycobacterium chimaera infection after open-chest heart surgery. Clin Infect Dis 2015;61:67-75.

6 Gotting T, Klassen S, Jonas D et al. Heater-cooler units: contamination of crucial devices in cardiothoracic surgery. J Hosp Infect 2016;93:223-8

7 Centers for Disease Control and Prevention. CDC has updated the notification toolkit to include frequently asked questions for hospitals notifying patients of risk from contaminated heater-cooler devices used during cardiac surgery. www.cdc.gov/hai/outbreaks/heatercooler.html [Accessed 20 June 2018].
8 Butterworth J. Mycobacterium chimaera associated haemophagocytic lymphohistiocytosis. Open Journal of Blood Diseases 2016:6:53-8.

Address for correspondence: Dr Shailesh Dalvi, Glan Clwyd Hospital, Cardiology, Rhyl LL18 5U], UK.

Email: drdalvi75@yahoo.com 